\title{
Urban Heat Islands and Vegetation Cover as a Controlling Factor
}

\author{
Manawadu L. ${ }^{1}$ and Ranagalage M. ${ }^{2 *}$ \\ ${ }^{1}$ Department of Geography, University of Colombo, Colombo 03, Sri Lanka \\ ${ }^{2}$ Department of Social Sciences, Rajarata University of Sri Lanka, Sri Lanka \\ *manjularanagalage@gmail.com
}

\begin{abstract}
Cities play an important role in climate change mitigation and adaptation and enhancing climate resilience of their slum and vulnerable residents. Climate change adds to existing challenges faced by cities. Climate change, together with a decrease in absorption capacity of greenhouse gasses due reduction in the amount of green cover, parks, trees and agricultural surfaces in urban areas, poses serious threats to urban infrastructure, access to basic services and quality of life in cities and negatively affect the urban economy. At the same time, rapid urban growth, growing urban poverty and increasing food prices raise concerns about urban food security, especially for the poor. Cities are highly vulnerable to disruption in critical (food) supplies and climate change exacerbates this vulnerability.

The main objective of this study is to examine the spatial pattern of surface temperature in the Kesbewa Urban Council Area and temperature variation with the different UPAF (Urban Peri-urban and Agriculture and Forestry) regions using space born techniques and ground verifications.

GIS and Remote Sensing techniques were the main analytical tools of this study in data collection as well as data processing and analysis. Landsat ETM+ image from 2003 used to extract the surface temperature layer and model has verified using air temperature data collected throughout the KUC area for the last five months period using 22 HOBO meters installed in different UPAF regions in the city. UPAF regions have identified and demarcated using Google image and visually interpreted with the field experiences.
\end{abstract}

The surface temperature model developed using satellite images is positively correlated with the ground temperature data collected and results revealed that there is a very positive relationship between the UPAF regions and the surface temperature.

Keywords: Urban peri-urban and agriculture and forestry, Urban heat islands, Vegetation cover 